\title{
Distribuição do fluxo de ar em silos com sistema de aeração: Análise por elementos finitos ${ }^{1}$
}

Ivano A. Devilla², Sandra M. Couto ${ }^{3} \&$ Daniel M. de Queiroz ${ }^{3}$

\author{
1 Parte da Tese de Doutorado do primeiro autor \\ 2 Universidade Estadual de Goiás - UNuCET. BR 153 km 98, CEP 75000-000, Anápolis,GO. Fone: (062) 324-2176. \\ E-mail: devilla@ueg.br (Foto) \\ ${ }^{3}$ DEA/UFV. Av. Ph Rolfs s/nº, Viçosa,MG. Fone: (031) 3899-1927. E-mail: scouto@ufv.br; Fone: (031) $3891-2270$. \\ E-mail: daniel@ufv.br
}

Protocolo 38 - 24/2/2003 - Aprovado em 28/10/2004

\begin{abstract}
Resumo: A distribuição de ar em uma massa de grãos de milho submetida a um processo de aeração, foi obtida, neste trabalho, através de simulações em que se empregou a técnica de elementos finitos. $O$ produto ( $13 \%$ b.u.) encontrava-se armazenado em silo metálico provido de dutos circulares para aeração. A massa de grãos foi considerada um conjunto de regiões (resistências distribuídas) que oferecem diferentes resistências ao escoamento de ar nas direções horizontal e vertical (anisotropia). O modelo para distribuição de ar no silo foi validado por meio de experimentos, usando-se condições idênticas às simuladas com um fluxo de ar de $0,0157 \mathrm{~m}^{3} \mathrm{~s}^{-1} \mathrm{~m}^{-2}$. A distribuição de ar proveniente das simulações, indicou a existência de zonas de baixa velocidade na massa de grãos situadas, na sua parte inferior, próximas ao centro do silo e à sua parede. As velocidades do ar na superfície da massa de grãos, simuladas e experimentais, tenderam a ser uniformes. A utilização da técnica de elementos finitos para a predição da distribuição do fluxo de ar, em silos providos de sistema de aeração, mostrou-se satisfatória e envolveu um erro relativo médio de $6,9 \%$.
\end{abstract}

Palavras-chave: aeração, elementos finitos, fluxo de ar, milho

\section{Airflow distribution in aerated silos: finite element analysis}

\begin{abstract}
In this work the finite element technique was used to obtain the air distribution in a mass of corn kernels submitted to an aeration process. The product $(13 \% \mathrm{w} . \mathrm{b}$.) was stored in metallic bin provided with a circular duct aeration system. The mass of grains was considered as being composed of regions (distributed resistances) that offer different resistances to the airflow in the horizontal and vertical directions. The model for air distribution in the bin was validated through experiments under similar conditions to the simulated ones with $0.0157 \mathrm{~m}^{3} \mathrm{~s}^{-1} \mathrm{~m}^{-2}$ airflow. The simulated air distribution indicated the existence of zones of low air velocities in the mass of grains located at the base of the bin (close to the center and to the wall of the bin). The simulated and experimental air velocities on the surface of the mass of grains showed a uniform tendency. The use of the finite element technique for the prediction of the airflow distribution in bins with aeration system was satisfactory and it involved a mean relative error of $6.9 \%$.
\end{abstract}

Key words: aeration, finite element, airflow, corn

\section{INTRODUÇÃO}

Além do esforço para aumentar a produção de alimentos, necessariamente há que se incrementar as condições para uma armazenagem segura dos grãos; para tal, é conveniente que os grãos estejam secos e, quando preciso, sejam ventilados durante o armazenamento. Nesses procedimentos, o ar é forçado por um ventilador, a escoar através da massa de grãos.
A deterioração de grãos armazenados, que é altamente dependente da temperatura e da umidade dos grãos, está relacionada com a respiração do produto e de microrganismos que o acompanham (Sauer, 1992). Por outro lado, o uso adequado da aeração em uma massa de grãos armazenada é de fundamental importância para se manter a qualidade do produto, além de reduzir o custo da armazenagem, enquanto a temperatura 
e o teor de umidade podem ser reduzidos com a aeração, tendose por consequiência menor deterioração dos grãos.

O sucesso dos processos de secagem e aeração depende da uniformidade de distribuição do ar no interior da massa de grãos que, por sua vez, depende da resistência que o produto oferece à passagem do fluido (Haque et al., 1982; Sokhansanj et al., 1990; Yang \& Williams, 1990; Alagusundaram et al., 1994).

A má distribuição do ar em uma massa de grãos armazenados pode provocar o desenvolvimento de fungos em áreas onde a velocidade do ar é baixa e, também, ocasionar a supersecagem dos grãos em áreas com alta velocidade do ar.

A resistência dos grãos e das sementes ao escoamento do ar tem sido estudada por vários cientistas (Shedd, 1951; Hukill \& Ives, 1955; Jayas et al., 1987; Giner et al., 1996). Alagusundaram \& Jayas (1990) apresentaram uma revisão sobre as várias equações utilizadas para determinar a resistência ao fluxo de ar oferecida por produtos agrícolas consultaram que vários trabalhos assumem a massa de grãos como um meio isotrópico, porém relatos encontrados na literatura mostram que a massa de grãos deve ser considerada um meio anisotrópico (Kumar \& Muir, 1986; Jayas et al., 1987; Kay et al., 1989; Sokhansanj et al.,1990).

A distribuição do fluxo de ar em grãos armazenados em silos dotados de sistema de aeração, tem sido modelada por Miketinac et al. (1986), Sinício et al. (1992) e Thorpe (2002) como um problema bidimensional, e por Smith (1982) e Jayas \& Muir (1991) como problema tridimensional.

Técnicas numéricas têm sido amplamente usadas na resolução de problemas de escoamento de fluidos; dentre estas, a técnica de elementos finitos vem sendo utilizada com sucesso por vários pesquisadores, para a resolução de diferentes problemas envolvendo escoamentos, por exemplo: Rojano et al. (1998); Ferguson (1995); Gong \& Mujumdar (1995); Lai(1980).

Segundo Marchant (1976) o método de elementos finitos pode ser usado com razoável precisão, para resolver equações que descrevem a distribuição do fluxo de ar em produtos agrícolas. O método, em comparação com as outras técnicas, possui a vantagem de resolver as equações para sistemas em qualquer forma geométrica.

Miketinac et al. (1986) determinaram, através da técnica de elementos finitos, a distribuição do fluxo de ar em silos de fundo plano e cônicos e com diferentes dutos de distribuição do ar, e constataram que a técnica de elementos finitos pode ser usada para prever a distribuição do ar nos silos e determinar as zonas onde ocorre baixa velocidade do ar.

O problema de escoamento de fluidos é definido pelas leis de conservação de massa, de momentum e de energia. Quando se consideram as seguintes restrições: (1) o fluido é newtoniano, (2) o fluido é incompressível, (3) não ocorre mudança de fase, (4) o escoamento é isotérmico, (5) o escoamento é permanente e (6) o domínio espacial do problema não muda com o tempo, as equações de conservação podem ser expressas como (Andrade et al., 2001)

\section{Conservação de massa}

$$
\frac{\partial V_{x}}{\partial x}+\frac{\partial V_{y}}{\partial y}+\frac{\partial V_{z}}{\partial z}=0
$$

em que:

$\vec{V}-\left(V_{x}, V_{y}\right.$ e $\left.V_{z}\right)$ - componentes do vetor velocidade do fluido nas direções $\mathrm{x}$, y e $\mathrm{z}$, respectivamente

$\mathrm{x}, \mathrm{y} \mathrm{e} \mathrm{z}$ - coordenadas cartesianas

\section{Equações de momentum}

$$
\begin{gathered}
\frac{\partial\left(\rho \mathrm{V}_{\mathrm{x}} \mathrm{V}_{\mathrm{x}}\right)}{\partial \mathrm{x}}+\frac{\partial\left(\rho \mathrm{V}_{\mathrm{y}} \mathrm{V}_{\mathrm{x}}\right)}{\partial \mathrm{y}}+\frac{\partial\left(\rho \mathrm{V}_{\mathrm{z}} \mathrm{V}_{\mathrm{x}}\right)}{\partial \mathrm{z}}=\rho \mathrm{g}_{\mathrm{x}}-\frac{\partial \mathrm{P}}{\partial \mathrm{x}}+ \\
\mathrm{R}_{\mathrm{x}}+\frac{\partial}{\partial \mathrm{x}}\left(\mu_{\mathrm{e}} \frac{\partial \mathrm{V}_{\mathrm{x}}}{\partial \mathrm{x}}\right)+\frac{\partial}{\partial \mathrm{y}}\left(\mu_{\mathrm{e}} \frac{\partial \mathrm{V}_{\mathrm{x}}}{\partial \mathrm{y}}\right)+\frac{\partial}{\partial \mathrm{z}}\left(\mu_{\mathrm{e}} \frac{\partial \mathrm{V}_{\mathrm{x}}}{\partial \mathrm{z}}\right) \\
\frac{\partial\left(\rho \mathrm{V}_{\mathrm{x}} \mathrm{V}_{\mathrm{y}}\right)}{\partial \mathrm{x}}+\frac{\partial\left(\rho \mathrm{V}_{\mathrm{y}} \mathrm{V}_{\mathrm{y}}\right)}{\partial \mathrm{y}}+\frac{\partial\left(\rho \mathrm{V}_{\mathrm{z}} \mathrm{V}_{\mathrm{y}}\right)}{\partial \mathrm{z}}=\rho \mathrm{g}_{\mathrm{y}}-\frac{\partial \mathrm{P}}{\partial \mathrm{y}}+ \\
\frac{\partial\left(\rho \mathrm{V}_{\mathrm{x}} \mathrm{V}_{\mathrm{z}}\right)}{\partial \mathrm{x}}+\frac{\partial\left(\rho \mathrm{V}_{\mathrm{y}} \mathrm{V}_{\mathrm{z}}\right)}{\partial \mathrm{y}}+\frac{\partial\left(\rho \mathrm{V}_{\mathrm{z}} \mathrm{V}_{\mathrm{z}}\right)}{\partial \mathrm{z}}=\rho \mathrm{g}_{\mathrm{z}}-\frac{\partial \mathrm{P}}{\partial \mathrm{z}}+ \\
\mathrm{R}_{\mathrm{z}}+\frac{\partial}{\partial \mathrm{x}}\left(\mu_{\mathrm{e}} \frac{\partial \mathrm{V}_{\mathrm{z}}}{\partial \mathrm{x}}\right)+\frac{\partial}{\partial \mathrm{y}}\left(\mu_{\mathrm{e}} \frac{\partial \mathrm{V}_{\mathrm{y}}}{\partial \mathrm{y}}\right)+\frac{\partial}{\partial \mathrm{z}}\left(\mu_{\mathrm{e}} \frac{\partial \mathrm{V}_{\mathrm{z}}}{\partial \mathrm{z}}\right)+\frac{\partial}{\partial \mathrm{z}}\left(\mu_{\mathrm{e}} \frac{\partial \mathrm{V}_{\mathrm{z}}}{\partial \mathrm{z}}\right)
\end{gathered}
$$

em que:

$P$ - pressão do fluido

$\rho$ - densidade do fluido

$\mathrm{g}-\left(\mathrm{g}_{\mathrm{x}}, \mathrm{g}_{\mathrm{y}}\right.$ e $\left.\mathrm{g}_{\mathrm{z}}\right)$ - componentes da aceleração da gravidade nas direções $\mathrm{x}, \mathrm{y}$ e $\mathrm{z}$, respectivamente

$\mathrm{R}$ - $\left(\mathrm{R}_{\mathrm{x}}, \mathrm{R}_{\mathrm{y}} \mathrm{e} \mathrm{R}_{\mathrm{z}}\right)$ - representam quaisquer termos adicionais (fontes ou sorvedouros) nas direções $\mathrm{x}, \mathrm{y} \mathrm{e} \mathrm{z}$, respectivamente $\mu_{e}$ - viscosidade efetiva

No método dos elementos finitos, aplicado a escoamentos de fluidos, a região espacial em estudo é dividida em elementos conectados por pontos comuns (nós), e as Eqs. (1), (2), (3) e (4) são discretizadas para cada elemento. O método de Garlekin, o dos resíduos ponderados (Segerlind, 1984), é usado na obtenção de matrizes para os elementos, que são acopladas em uma única matriz. O sistema de equações resultante é resolvido para cada grau de liberdade obtendo-se, assim, valores de pressão e de velocidade em cada nó do sistema.

Os termos $R_{X}, R_{y}$ e $R_{Z}$, nas Eqs. (2), (3) e (4), podem ser considerados resistências distribuídas (representações macroscópicas de geometria no interior da região em estudo) e são uma maneira conveniente de se aproximar efeitos de meios porosos sem realmente modelar a sua geometria.

O presente trabalho foi desenvolvido com o objetivo de modelar, usando-se a técnica de elementos finitos, a distribuição 
de ar em grãos de milho, considerados meio anisotrópico, armazenados em silos metálicos providos de sistema de ventilação.

\section{MATERIAL E MÉTODOS}

\section{Sistema modelado}

O sistema modelado esquematizado na Figura 1A, consistiu de uma massa de grãos de milho ( $13 \%$ b.u.) contida, até a altura de 1,6 m, em um silo metálico cilíndrico de $1,8 \mathrm{~m}$ de raio. Escoamento de ar foi introduzido na parte inferior do sistema, através de uma abertura que simulava um duto circular perfurado (largura de 0,2 m), cuja vista superior é esquematizada na Figura 1B. O duto foi posicionado a uma distância radial de $1,1 \mathrm{~m}$ do eixo de simetria.

\section{Análise por elementos finitos}

O método dos elementos finitos foi usado para o desenvolvimento de um programa computacional, visando-se simular a distribuição do fluxo de ar no sistema (massa de grãos) provido de aeração. Para tal, utilizou-se o programa de modelagem por elementos finitos ANSYS, versão 5.7 (Ansys, 2001).

O sistema foi considerado simétrico em relação ao eixo longitudinal central do silo, eixo Y; assim, na análise do escoamento de ar no meio poroso considerou-se apenas a metade da seção longitudinal da massa de grãos. O sistema foi discretizado em elementos bidimensionais do tipo FLUID141 com 4 nós, que sofreram, durante a análise, rotação em torno do eixo de simetria. O leito de grãos de milho foi considerado

A.

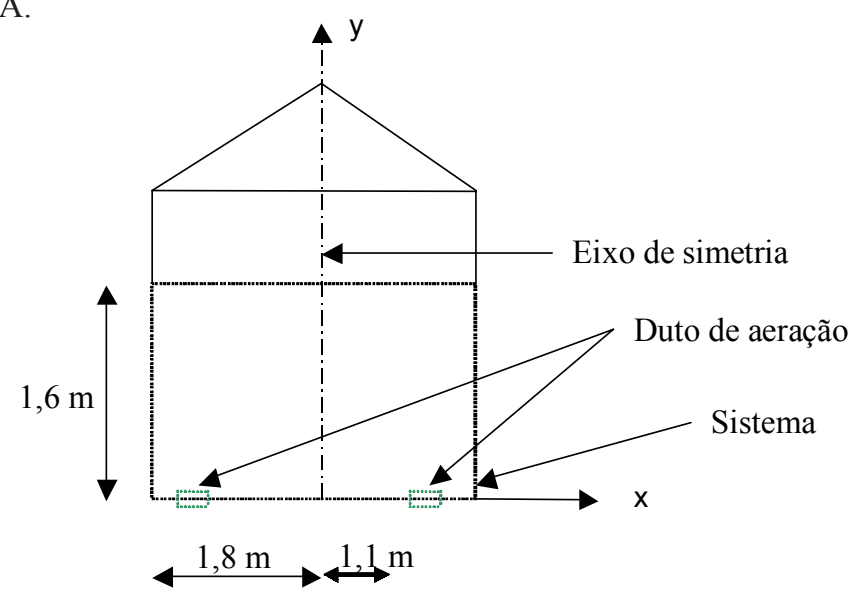

B.

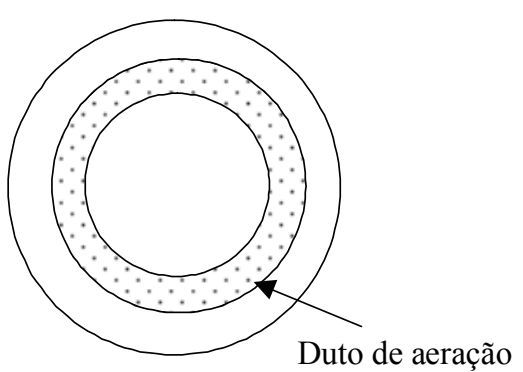

Figura 1. (A) Esquema do sistema estudado e da condição de simetria para modelagem do fluxo de ar e (B) vista superior do duto de aeração uma resistência distribuída. O tipo de elemento usado permite a introdução de constantes, representativas de resistências distribuídas no seu interior, nas direções X (RDx) e Y (RDy). Nas simulações, o sistema foi submetido a um fluxo de ar de $0,0157 \mathrm{~m}^{3} \mathrm{~s}^{-1} \mathrm{~m}^{-2}$ quadrado de área perfurada.

Valores das resistências RDx e RDy foram obtidos através de um processo de otimização, descrito em Andrade (2001). Os valores (pressão estática versus profundidade), utilizados no processo de otimização, foram obtidos pela Eq. 5, sugerida por ASAE (2000), para a faixa de velocidade de $0,00025 \mathrm{a} 0,0168 \mathrm{~m} \mathrm{~s}^{-1}$. A queda de pressão, na direção X, foi estimada em 45\% da queda de pressão na direção Y, de acordo com Kay et al. (1989).

$$
\frac{\Delta \mathrm{P}}{\mathrm{L}}=\frac{\mathrm{aQ}^{2}}{\ln (1+\mathrm{bQ})}
$$

em que:

$\Delta \mathrm{P} \quad$ - perda de carga, $\mathrm{Pa}$

L - altura da camada, $m$

a e b - constantes que dependem do produto (para milho $a=9770$ e $b=8,55)$

Q - fluxo de ar, $\mathrm{m}^{3} \mathrm{~s}^{-1} \mathrm{~m}^{2}$

Os dados de entrada necessários à simulação da distribuição do fluxo de ar na massa de grãos, foram: geometria do sistema, propriedades do ar e valores das resistências distribuídas (RDx, RDy). Os contornos do sistema foram submetidos às seguintes condições: (a) velocidades nulas (direções X e Y) nas paredes, (b) velocidade (conhecida na direção Y) na abertura representando o duto de aeração, (c) velocidade nula (direção X) no eixo de simetria, e (d) pressão nula na extremidade superior do sistema (referência: $\mathrm{P}=1 \mathrm{~atm}$ ).

Na resolução do problema as considerações seguintes foram feitas: (a) regime permanente; (b) escoamento turbulento; (c) propriedades constantes (ar e partículas); (d) análise tridimensional; (e) os grãos de milho são tratados como resistência distribuída; (f) meio anisotrópico; (g) efeitos térmicos desprezíveis e $(\mathrm{h})$ fricção nas paredes do silo desprezível.

\section{Validação do modelo}

Um silo metálico cilíndrico de 3,6 m de diâmetro e 2,2 m de altura, com fundo totalmente perfurado, foi utilizado para validação do modelo. Um sistema de aeração, consistindo de um ventilador centrífugo com motor trifásico de $1,5 \mathrm{~kW}$ e um diafragma para controle da entrada do ar no ventilador, foi adaptado ao silo.

O fundo do silo perfurado foi coberto por chapas metálicas, de maneira que a área para a entrada de ar fosse em forma de anel circular, como esquematizado na Figura $1 \mathrm{~B}$.

O silo metálico foi carregado com milho a $13 \%$ b.u., até a altura de 1,6 m. O carregamento foi realizado com o auxílio de uma rosca transportadora, a qual descarregava o produto de forma excêntrica, e a altura de queda do produto foi de $2,3 \mathrm{~m}$. A superfície da massa de grãos foi nivelada para evitar possíveis caminhos preferenciais do ar.

Dois testes, cada um com duração de cinco dias, com um fluxo de ar de aeração de $0,0157 \mathrm{~m}^{3} \mathrm{~s}^{-1} \mathrm{~m}^{-2}$, foram realizados 
visando à validação do modelo. Diariamente, as velocidades do ar em vários pontos da superfície da massa de grãos, Figura 2, foram medidas. Os pontos situados nas linhas principais encontravam-se a distâncias radiais, a partir do centro do silo, de $0,3,0,9$ e $1,5 \mathrm{~m}$, e os pontos nos quadrantes a partir do centro do silo, a distâncias radiais de 0,4 e 1,6 m.

As velocidades foram medidas por meio de um anemômetro de pás rotatórias, com faixa de leitura de 0 a $35 \mathrm{~m} \mathrm{~s}^{-1}$ e erro de $\pm 2 \%$, devidamente calibrado. Para possibilitar a medida de velocidades baixas do ar, utilizou-se um dispositivo metálico com forma de um tronco de cone, o qual foi colocado na superfície da massa. O dispositivo possuía altura de $0,45 \mathrm{~m}$ e suas aberturas, inferior e superior, tinham diâmetros de $0,40 \mathrm{e}$ $0,0254 \mathrm{~m}$, respectivamente. A velocidade na superfície da massa de grãos foi calculada com base no princípio da conservação da massa.

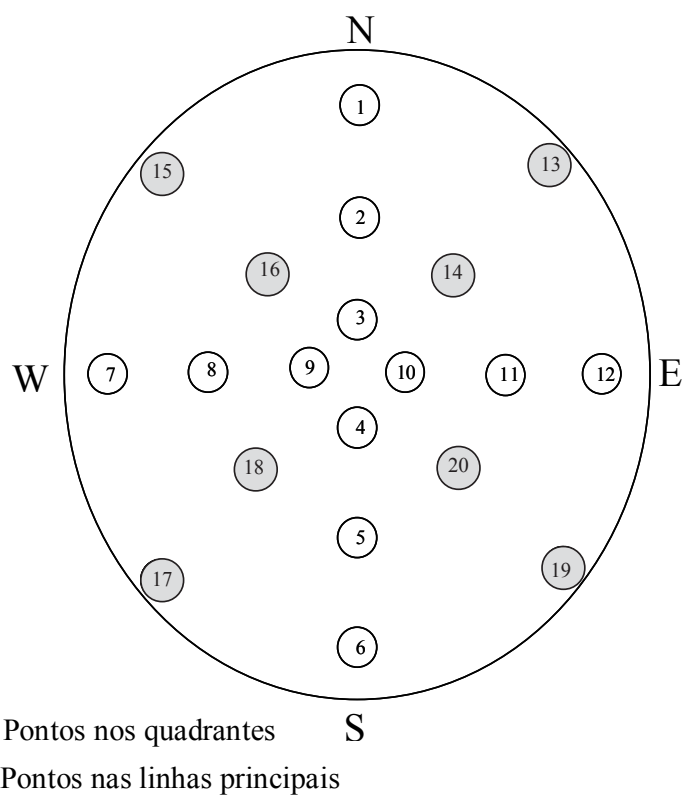

Figura 2. Esquema dos pontos de medição da velocidade do ar na massa de grãos

\section{RESULTADOS E DISCUSSÃO}

O sistema investigado foi discretizado, como mostrado na Figura 3, em 1.550 elementos, resultando em 1.632 nós.

Aos valores obtidos para as resistências distribuídas em função da velocidade do ar, após o processo de otimização, foram ajustadas equações polinomiais de primeiro grau, cujos coeficientes são apresentados na Tabela 1, acompanhados dos respectivos coeficientes de determinação $\left(\mathrm{R}^{2}\right)$.

Inicialmente, atribuíram-se, aos elementos situados sobre o duto de aeração, valores de resistência distribuída, máxima na direção Y e mínima na direção $\mathrm{X}$, devido ao escoamento de ar na saída do duto de aeração, que provoca a maior perda de carga na direção Y; aos demais elementos, deram-se-lhes valores médios de resistência nas duas direções. O programa computacional foi, então, executado, e as distribuições de

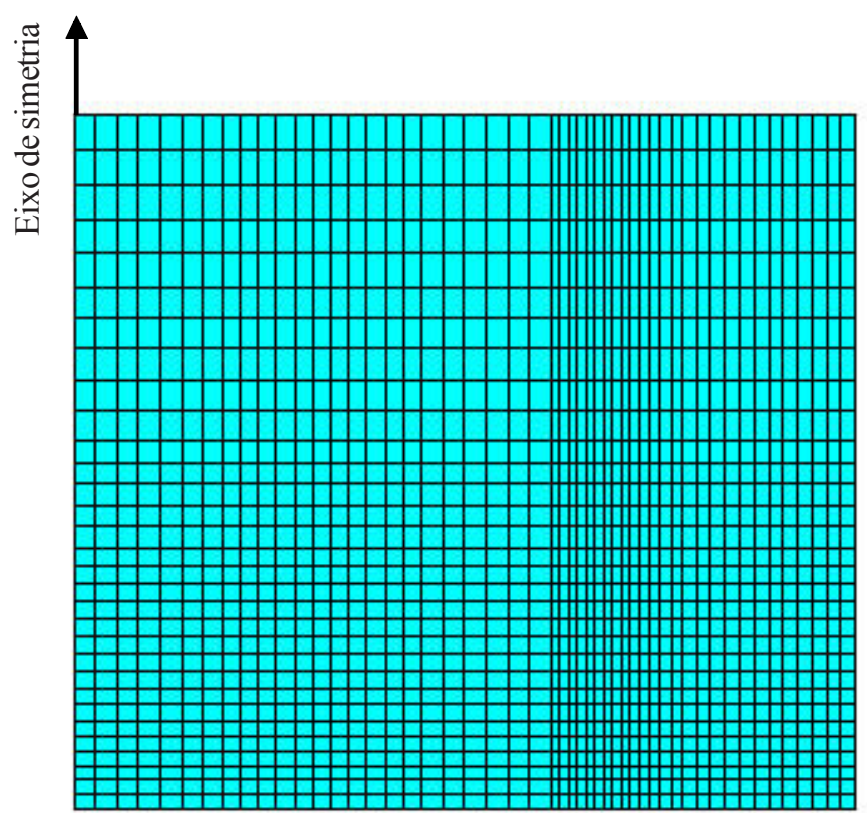

Figura 3. Malha para análise por elementos finitos

Tabela 1. Coeficientes das equações ajustadas $(\mathrm{RD}=\mathrm{A}+\mathrm{B} \mathrm{V})$ para expressar a resistência distribuída $(\mathrm{RD})$ em função da velocidade do ar (V)

\begin{tabular}{crcc} 
Resistência distribuída & A & B & $\mathrm{R}^{2}$ \\
\hline RDx & 28391986 & $117051172 *$ & 0,998 \\
RDy & 63057219 & $261521190 *$ & 0,999 \\
\hline * Significativo a 1\% de probabilidade, pelo teste t. & &
\end{tabular}

velocidade nas direções $\mathrm{X}$ e $\mathrm{Y}$ foram determinadas.

Os resultados obtidos (Figuras 4A e B) nesse primeiro procedimento, foram usados como guia para a divisão do sistema em diferentes regiões, conforme apresentado na Figura 5. Consideram-se, como critério para a divisão das regiões, as faixas de velocidade. Um processo iterativo foi implementado com o objetivo de determinar valores adequados de resistências distribuídas para cada região. Este processo consistiu das seguintes etapas: (1) obter a velocidade média do ar, nas direções X e Y, em cada região; (2) usando-se esses valores de velocidade, determinar as resistências distribuídas (RDx e RDy) para cada região, através das equações expostas na Tabela 1; (3) introduzir no sistema os novos valores de resistência, executar o programa computacional e obter a nova distribuição de velocidade nas direções $\mathrm{X}$ e Y e (5) comparar a nova distribuição de velocidades com aquela já obtida anteriormente. Se a diferença entre as velocidades for maior que $\left|1 \times 10^{-5}\right| \mathrm{m} \mathrm{s}^{-1}$, retornar à etapa 1 do processo; caso contrário, os valores atribuídos às resistências distribuídas de cada região, serão considerados os mais prováveis.

Os valores usados para as resistências distribuídas nas direções $\mathrm{X}$ e Y, representativas do meio poroso, para cada região, são apresentados na Tabela 2.

A distribuição do vetor velocidade do ar, obtida pela análise do escoamento de ar no sistema usando-se a técnica de elementos finitos, quando o sistema foi submetido ao fluxo de 


\section{A. Devilla et al.}

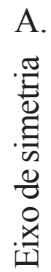
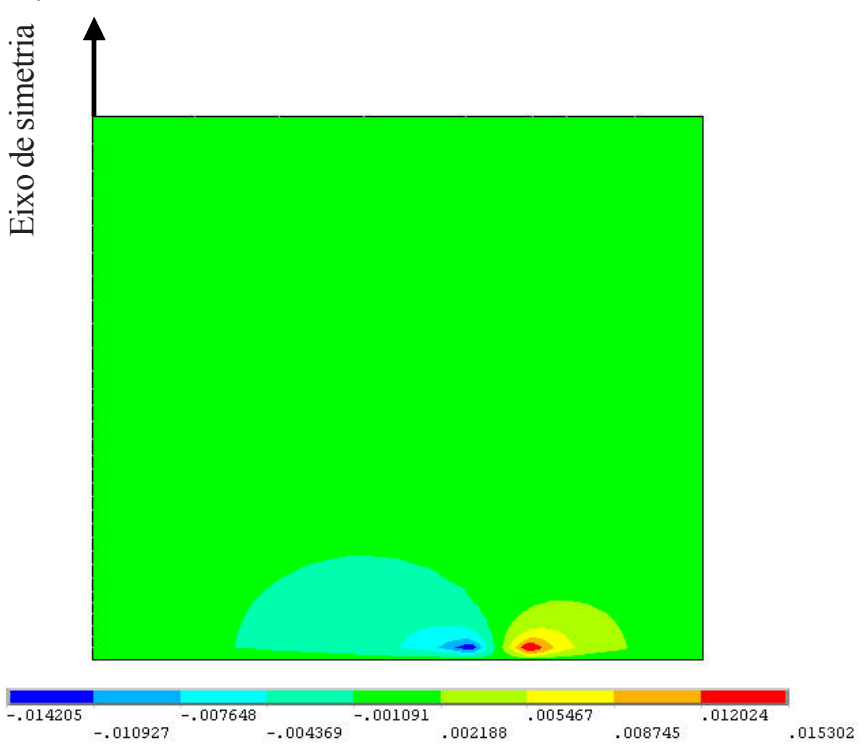

B.

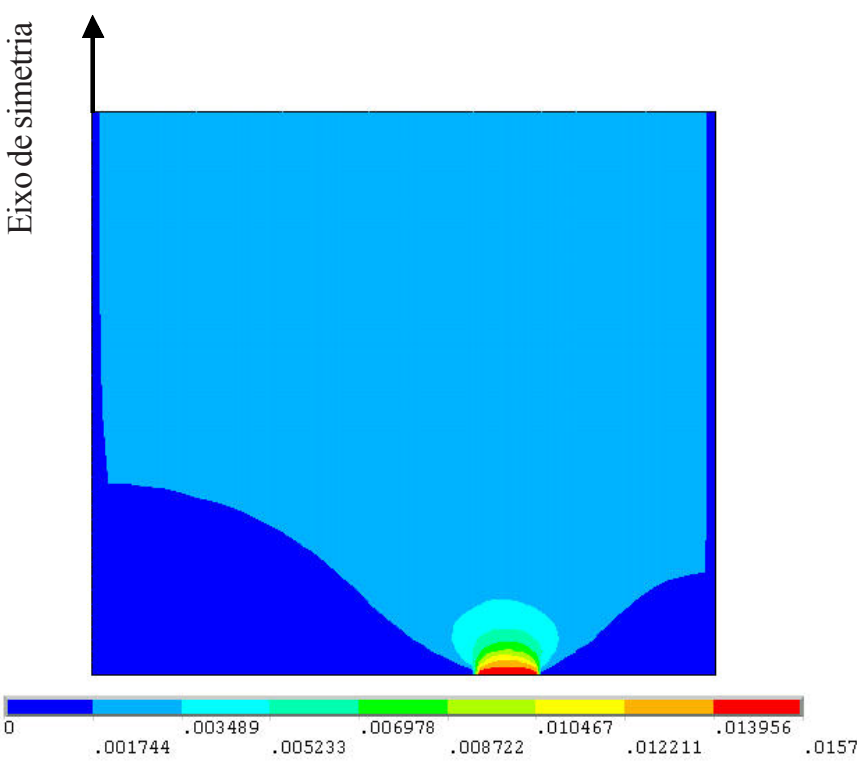

Figura 4. Distribuição de velocidades na direção X(A) Y (B)

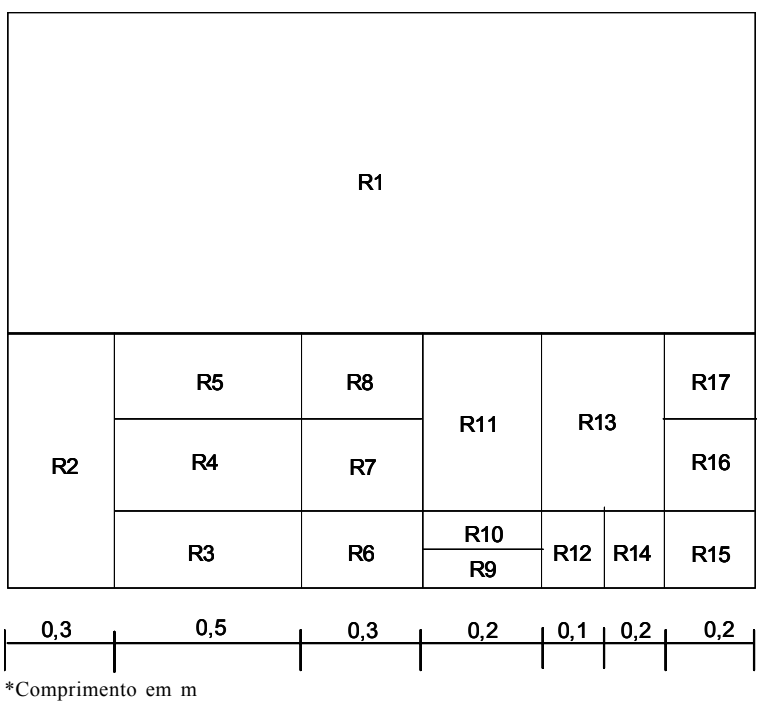

Figura 5. Esquema da divisão do sistema em regiões
Tabela 2. Resistências distribuídas (RD) nas direções X e Y em diferentes regiões do sistema modelado

\begin{tabular}{ccc}
\hline \multirow{2}{*}{ Região } & $\mathrm{RDx}$ & $\mathrm{RDy}$ \\
\cline { 2 - 3 } & $\mathrm{x} 10^{7}\left(\mathrm{~m}^{-2}\right)$ & $\mathrm{x} 10^{7}\left(\mathrm{~m}^{-2}\right)$ \\
\hline 1 & 2,8582 & 6,3741 \\
2 & 2,8582 & 6,3285 \\
3 & 2,8672 & 6,3285 \\
4 & 2,8612 & 6,3399 \\
5 & 2,8582 & 6,3627 \\
6 & 2,8892 & 6,3665 \\
7 & 2,8612 & 6,3741 \\
8 & 2,8582 & 6,3741 \\
9 & 2,9340 & 6,6023 \\
10 & 2,8676 & 6,4198 \\
11 & 2,8582 & 6,3741 \\
12 & 2,9054 & 6,3741 \\
13 & 2,8582 & 6,3741 \\
14 & 2,8776 & 6,3399 \\
15 & 2,8582 & 6,3285 \\
16 & 2,8582 & 6,3627 \\
17 & 2,8582 & 6,3741 \\
\hline
\end{tabular}

ar de $0,0157 \mathrm{~m}^{3} \mathrm{~s}^{-1} \mathrm{~m}^{-2}$, é mostrada na Figura 6. Verifica-se que, no fundo do silo, próximo à sua parede e ao eixo de simetria, há áreas onde a velocidade do ar é muito baixa, proporcionando zonas com pouca aeração. Este fato é preocupante, pois nessas zonas o baixo fluxo de ar pode dificultar o resfriamento dos grãos. Observa-se, ainda, uma distribuição uniforme da velocidade do ar, na superfície da massa de grãos.

Quando o sistema foi submetido ao fluxo $0,0157 \mathrm{~m}^{3} \mathrm{~s}^{-1}$ por $\mathrm{m}^{-2}$ de área perfurada, constatou-se que na região vizinha ao duto havia vetores velocidade, cujos módulos eram superiores a $0,0157 \mathrm{~m}^{3} \mathrm{~s}^{-1} \mathrm{~m}^{-2}$, e que a direção desses vetores era predominantemente próxima da horizontal. Este fato se deve, provavelmente, à menor resistência oferecida pela massa de grãos nesta direção.

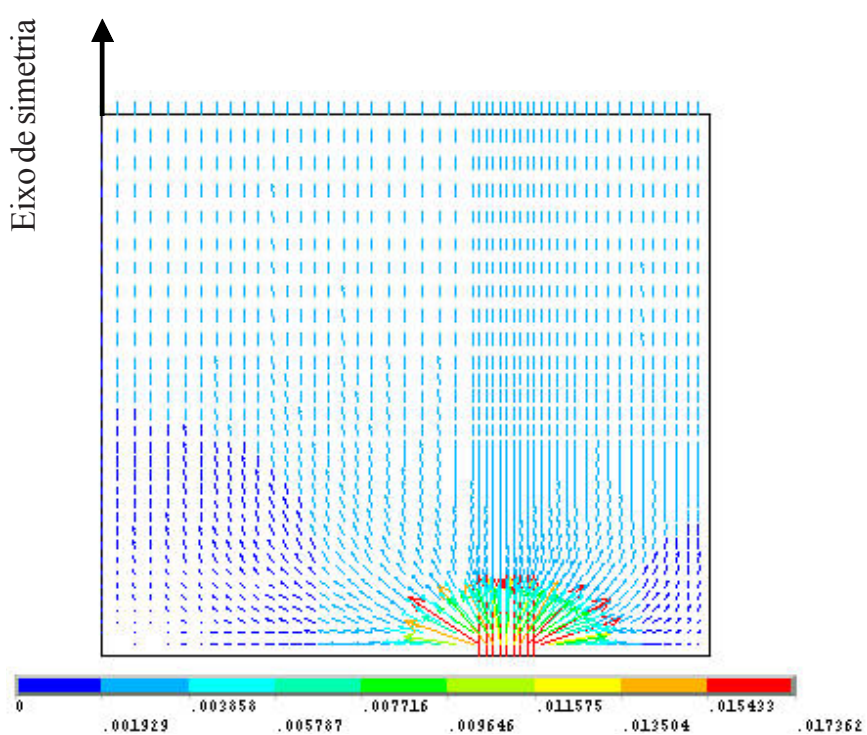

Figura 6. Vetor de velocidade do ar simulado para o fluxo de $0,0157 \mathrm{~m} \mathrm{~s}^{-1} \mathrm{~m}^{-2}$ 


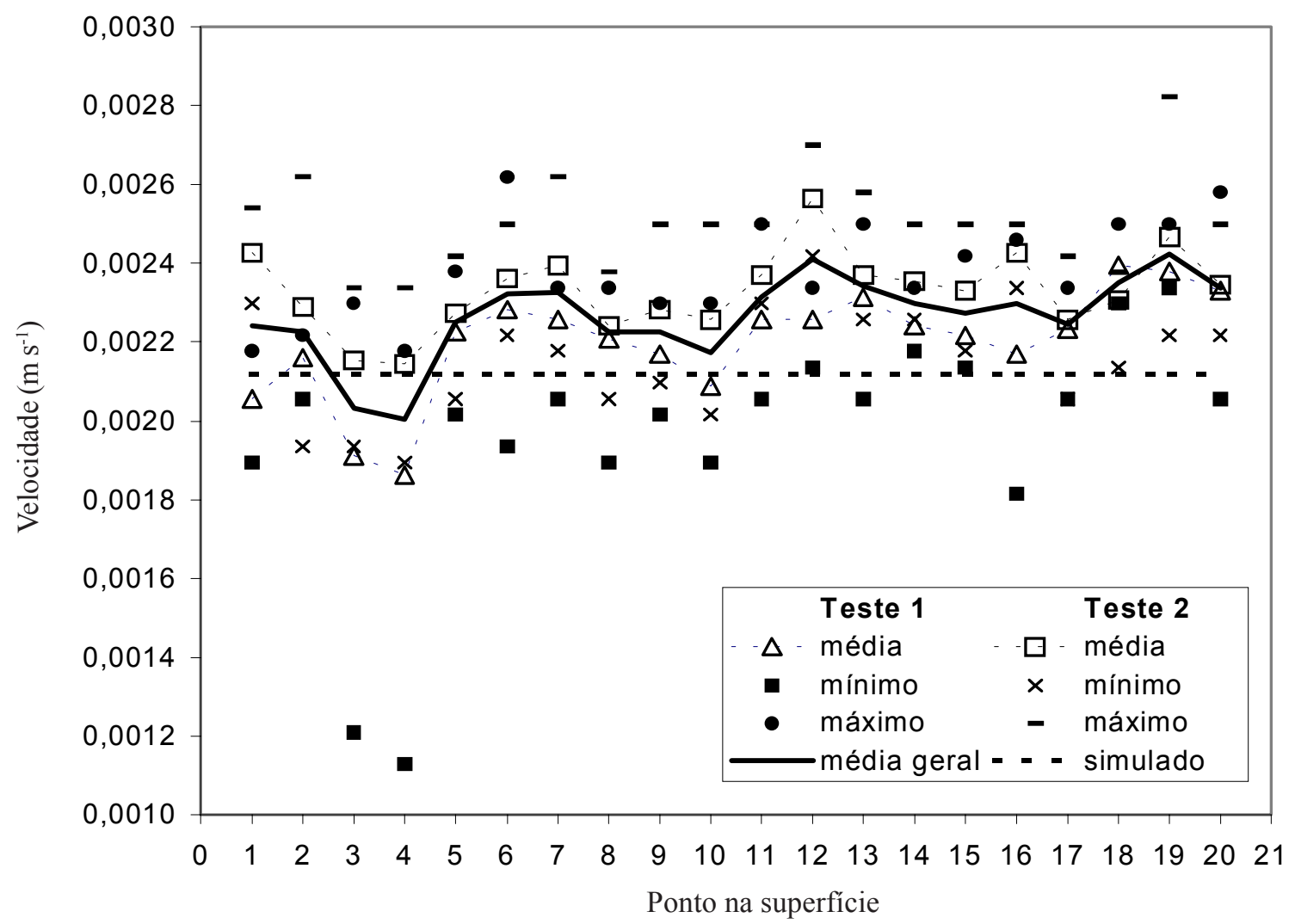

Figura 7. Resultados simulados e experimentais da velocidade nos dois testes de aeração

Os valores (médios, máximos e mínimos) das velocidades na superfície da massa de grãos, provenientes dos dois testes experimentais de aeração (cada um com duração de cinco dias), usando-se um fluxo de ar de $0,0157 \mathrm{~m}^{3} \mathrm{~s}^{-1} \mathrm{~m}^{-2}$, são apresentados na Figura 7, para cada um dos pontos da superfície dos grãos, conforme esquematizado na Figura 2.

Observa-se, na Figura 7, que na maioria dos pontos as menores velocidades ocorreram durante o teste 1. Quando se desconsideraram os pontos, os valores médios da velocidade do ar nos testes 1 e 2 foram iguais a $0,0022 \pm 0,0002$ e 0,0023 $0,0002 \mathrm{~m} \mathrm{~s}^{-1}$, respectivamente, e a média geral, nos dois testes, foi igual a $(0,0023 \pm 0,0002) \mathrm{m} \mathrm{s}^{-1}$. Quando se considerou a variação da velocidade em cada ponto, durante os cinco dias de teste, nos testes 1 e 2 , houve desvios máximos de 0,0004 e $0,0003 \mathrm{~m} \mathrm{~s}^{-1}$ e desvios médios de 0,0002 e 0,0001 , respectivamente, parecendo indicar que a variação da velocidade do ar na superfície da massa de grãos, durante os dias de aeração, foi maior ou equivalente à variação, devido à localização do ponto na superfície.

Encontram-se, na Figura 7, os valores simulados para as velocidades nos pontos da superfície, observando-se que a distribuição do ar na superfície da massa tendeu a ser uniforme $\left(0,00212 \mathrm{~m} \mathrm{~s}^{-1}\right)$. Os erros relativos envolvidos nas simulações das velocidades na superfície dos grãos, referindo-se aos valores médios experimentais como os mais corretos, são apresentados na Tabela 3 .

Verifica-se que os maiores erros de simulação ocorreram próximo à parede devido, provavelmente, à forma ondulada da parede (constituída por chapas corrugadas), que no modelo foi considerada reta, ou pela baixa velocidade do ar nesta localização.
Tabela 3. Erros relativos (ER) para cada ponto simulado

\begin{tabular}{cccccc}
\hline Ponto & ER (\%) & Localização & Ponto & ER (\%) & Localização \\
\hline 1 & 4,7 & Parede & 11 & 8,0 & Centro \\
2 & 4,3 & Centro & 12 & 11,4 & Parede \\
3 & 4,8 & Centro & 13 & 8,8 & Parede \\
4 & 6,3 & Centro & 14 & 7,7 & Centro \\
5 & 5,4 & Centro & 15 & 6,0 & Parede \\
6 & 8,0 & Parede & 16 & 7,7 & Centro \\
7 & 8,1 & Parede & 17 & 4,8 & Parede \\
8 & 4,3 & Centro & 18 & 9,8 & Centro \\
9 & 4,3 & Centro & 19 & 11,8 & Parede \\
10 & 2,0 & Centro & 20 & 9,3 & Centro \\
\hline
\end{tabular}

Nota-se, também, que os erros envolvidos na predição da distribuição de velocidade usando-se a técnica de elementos finitos, foram relativamente baixos e variaram de 2,0 a $11,8 \%$, com valor médio de $6,9 \%$. Esses erros são considerados aceitáveis diante das incertezas experimentais. Se a velocidade experimental fosse considerada a mesma em todos os pontos $\left(0,0023 \mathrm{~m} \mathrm{~s}^{-1}\right)$, o erro envolvido na simulação seria igual a 7,4\%.

Desta forma, vê-se que a técnica de elementos finitos pode ser utilizada como uma ferramenta importante na determinação da distribuição do ar em silos providos de sistema de aeração, dotados de dutos circulares.

\section{CONCLUSÕES}

1. A utilização da técnica de elementos finitos para a predição da distribuição do fluxo de ar, em silos providos de sistema de 
aeração, mostrou-se satisfatória. O erro relativo médio envolvido nas simulações foi de $6,9 \%$.

2. A distribuição de ar, proveniente das simulações, em silos dotados de dutos circulares para aeração, indica a existência de zonas de baixa velocidade na massa de grãos, situadas na sua parte inferior, próximas ao centro do silo e na parede.

3. A velocidade do ar na superfície da massa de grãos, oriunda da análise de escoamento de ar, tendeu a ser uniforme. Tendência similar aconteceu com as velocidades obtidas experimentalmente.

\section{LITERATURA CITADA}

Alagusundaram, K.; Jayas, D.S. Airflow resistance of grains and oilseeds. Postharvest News and Information, Amsterdam, v.1, n.4, p.279-283, 1990.

Alagusundaram, K.; Jayas, D.S.; Friesen, O.H.; White, N.D.G. Airflow patterns through wheat, barley, and canola in bins with partially perforated floors - an experimental investigation. Applied Engineering in Agriculture, St. Joseph, v. 10, n.6, p.791-796, 1994.

Andrade, E.T.; Couto, S.M.; Queiroz, D.M. Distribuição de pressão estática em uma coluna de canola: Análise por elementos finitos. Revista Brasileira de Engenharia Agrícola e Ambiental, Campina Grande, v.5, n.2, p.288 - 295, 2001.

ANSYS. ANSYS/Multiphysics product (Program and Program Documentation), Realease 5.7, Houston: ANSYS Inc, 2001.

ASAE - American Society of Agricultural Engineers. Agricultural Engineers Yearbook. St. Joseph: Michigan, 2000. 796 .

Ferguson, W.J. A control volume finite element numerical simulation of the high temperature drying of spruce. Drying Technology, New York, v.3, n.3, p.607-634, 1995.

Giner, S.A.; Denisienia, E. Pressure drop through wheat as affected by air velocity, moisture content and fines. Journal Agricultural Engineering Research, Silsoe, n.63, p.73-86, 1996.

Gong, Z.X.; Mujumdar, A.S. A two-dimensional finite element model for kiln-drying of refractory concrete. Drying Technology, New York, v.3, n.3, p.585-605, 1995.

Haque, E.; Ahmed, Y.N.; Deyoe, C.W. Static pressure drop in a fixed bed of grain as affected by grain moisture content. Transactions of the ASAE, St. Joseph, v.25, n.4, p.10951098, 1982.

Hukill, W.V.; Ives, N.C. Radial air flow resistence of grains. Agricultural Engineering, St. Joseph, v.36, n.5, p.332-335, 1955.

Jayas, D.S.; Muir, W.E. Airflow-pressure drop data for modeling fluid flow in anisotropic bulks. Transactions of the ASAE, St. Joseph, v.34, n.1, p.251 - 254, 1991.
Jayas, D.S.;. Sokhansanj, S.; Moysey, E.B.; Barber, E.M. The effect of airflow direction on the resistance of canola (Rapeseed) to airflow. Canadian Agricultural Engineering, Saskatoon, v.29, n.2, p.189-192, 1987.

Kay, R.L.; Bern, C.J.; Hurburgh Jr., C.R. Horizontal and vertical airflow resistance of shelled corn at various bulk densities. Transactions of the ASAE, St. Joseph, v.32, n.2, p.733-736, 1989.

Kumar, A.; Muir, W.E. Airflow resistance of wheat and barley affected by airflow direction, filling method and dockage. Transactions of the ASAE, St. Joseph, v.29, n.5, p.14231426, 1986.

Lai, F.S. Three-dimensional flow of air through non-uniform grain beds. Transactions of the ASAE, St. Joseph, v. 23, n. 2, p.729-734, 1980.

Marchant, J.A. The prediction of airflows in crop drying systems by the finite element method. Journal of Agricultural Engineering, Silsoe, v.21, p.417-429, 1976.

Miketinac, M.J.; Sokhansanj, S.; Jayas, D.S. Graphical analysis of airflow distribution in grain bins using the finite element method. Canadian Agricultural Engineering, Saskatoon, v.28, n.1, p.23-30, 1986

Rojano, A.; Ramirez, A.; Fitz, E.; Ojeda, W. Temperature distribution in a greenhouse due to shape factor, St. Joseph: ASAE, 1998. 10p. Paper No.98-4156.

Sauer, D.B. Storage of cereal grains and their products. Minnesota: American Association of Cereal Chemists, 1992. $614 p$.

Segerlind, L.J. Applied finite element analysis. New York: John Wiley, 1984.427p.

Shedd, C.K. Some new data on resistance of grains to air flow. Journal of Agricultural Engineering, Silsoe, v.32, n.9, p.493$495,520,1951$.

Sinicio, R.; Jayas, D.S.; Muir, W.E.; Sanderson, D.B. Finiteelement prediction of non-uniform airflow in fixed beds of wheat. Postharvest Biology and Technology, Amsterdam, v.2, p.51-59, 1992.

Smith, E.A. 3-Dimensional analysis of air velocity and pressure in beds of grain and hay. Journal Agricultural Engineering Research, Silsoe, v.27, p.101-117, 1982.

Sokhansanj, S.; Falacinski, A.A.; Sosulski, F.W.; Jayas, D.S.; Tang, J. Resistence of bulk lentils to airflow. Transactions of the ASAE, St. Joseph, v.33, n.4, p.1281-1285, 1990.

Thorpe, G. Airflow distribution in ventilated beds of grain. In: Navarro, S.; Noyes, R. The mechanics and physics of modern grain aeration management. Boca Raton: CRC Press, 2002. p.585-624

Yang, X.; Williams, D.L. Airflow resistence of grain sorghum as affected by bulk density. Transactions of the ASAE, St. Joseph, v.33, n. 6, p.1966-1970, 1990. 